\title{
Coherent diffraction imaging at space-group forbidden reflections
}

\author{
G. Beutier ${ }^{1}$, J. Eymery², M. Dupraz ${ }^{2,3}$, M.-I. Richard ${ }^{2,3}$, A. Wartelle ${ }^{1,3}$, M. Verdier ${ }^{1}$, M. de Boissieu ${ }^{1}$, S. P. Collins ${ }^{4}$, \\ E. Bellec ${ }^{3}$, V. Favre-Nicolin ${ }^{3}$, S. Leake ${ }^{3}$
}

\author{
${ }^{I}$ SIMaP, CNRS / Grenoble INP / Univ Grenoble Alpes, France, ${ }^{2}$ CEA, IRIG, France, ${ }^{3}$ ESRF, France, ${ }^{4}$ Diamond Light Source, United \\ Kingdom, \\ guillaume.beutier@grenoble-inp.fr
}

On one hand, coherent diffraction imaging (CDI) in Bragg geometry has emerged as a unique 3D microscopy of nanocrystals thanks to 3rd generation synchrotron sources. Away from absorption edges and at space-group allowed reflections, it provides not only the electronic density, but also, encoded in the phase, the atomic displacement field with respect to the mean lattice, which in turn reveals crystal strain, defects and domains [1-3]. On the other hand, some crystal structures have crystallographic reflections which are forbidden by the space-group symmetry but can nevertheless be observed at a suitable X-ray absorption edge, due to the anisotropy of the tensor of scattering (ATS) [4]. They are several orders of magnitude weaker than allowed reflections, but the absence of Thomson scattering allows the observation of various electronic phenomena related to electronic orders (magnetic, charge, orbital), static and dynamic atomic displacements.

The new generation of synchrotron sources, such as the ESRF "Extremely Bright Source", opens opportunities to perform CDI on such weak reflections. Here we report on the measurement of the (115) forbidden reflection of a GaN nanopillar at the Ga K edge. Sufficient statistics could be obtained in a total accumulation time of $\sim 30$ minutes for an entire rocking curve to retrieve the phase of the scattering function. Such measurement at high temperature would provide an image of the inhomogeneity of thermal motion in the crystal [5], which would be particularly interesting close to surfaces, inversion domain boundaries [3] and crystal defects. This proofof-principle experiment demonstrates that forbidden reflections are a new opportunity for CDI with the new synchrotron sources.
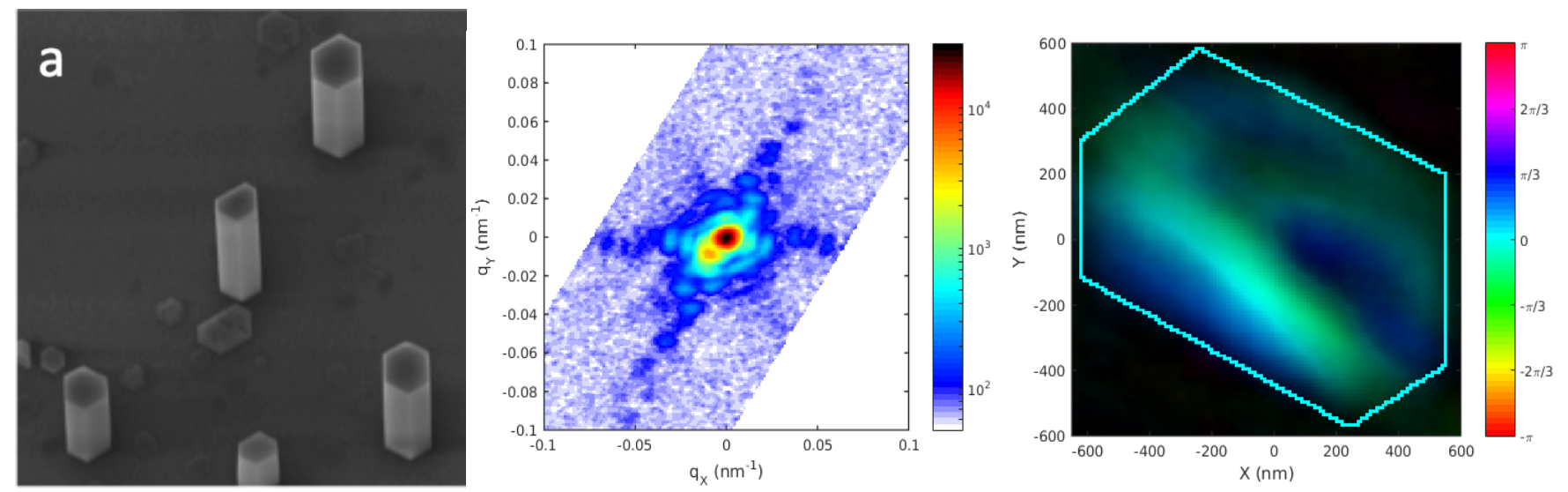

Figure 1. Left: electron microscopy of a typical area of the sample. Centre: (HK0) slice through the reciprocal space map around the (115) forbidden reflection. Right: 2D image of the complex scattering function in the basal plane as obtained from phase retrieval (the modulus and the phase are encoded by the brightness and the colour, respectively). The bright hexagon is a guide to the eye.

[1] Robinson, I. \& Harder, R. (2009). Nature Materials 8, 291.

[2] Clarke, J., Ihli, J., Schenk, A. S., Kim, Y.-Y., Kulak, A. N., Campbell, J. M., Nisbet, G., Meldrum, F. C. \& Robinson, I. K. (2015). Nature Materials 14, 780 .

[3] Labat, S., Richard, M.-I., Dupraz, M., Gailhanou, M., Beutier, G., Verdier, M., Mastropietro, F., Cornelius, T. W., Schülli, T. U., Eymery, J. \& Thomas, O. (2015). ACS Nano 9, 9210.

[4] Dmitrienko, V. E. (1983). Acta Cryst. A 39, 29.

[5] Beutier, G., Collins, S. P., Nisbet, G., Ovchinnikova, E. N. \& Dmitrienko, V. E. (2012). Eur. Phys. J. Special Topics $208,53$.

\section{Keywords: coherent diffraction imaging; forbidden reflection; resonant $X$-ray scattering}

The authors ackowledge the ESRF for beamtime allocation under project number MI-1377. 